\title{
Reconstruction of Velocity Distribution in Partially-Filled Pipe Based on Non-Uniform Under-Sampling
}

\author{
Yulin Jiang $\mathbb{D}^{1},{ }^{1}$ Qingmin Pu, ${ }^{1}$ and Wenbin Ding ${ }^{2}$ \\ ${ }^{1}$ College of Information Engineering, Zhongshan Polytechnic, Zhongshan 528400, China \\ ${ }^{2}$ College of Electronic and Engineering, Jiujiang University, Jiujiang 332000, China
}

Correspondence should be addressed to Yulin Jiang; ghostjiang@sina.com

Received 19 July 2019; Accepted 30 September 2019; Published 22 January 2020

Academic Editor: Mikhail Panfilov

Copyright (c) 2020 Yulin Jiang et al. This is an open access article distributed under the Creative Commons Attribution License, which permits unrestricted use, distribution, and reproduction in any medium, provided the original work is properly cited.

In the process of research on the flow velocity distribution in a partially filled pipe, the under-sampling of measurement data often occurs. For the first time, this problem is solved by the improved non-uniform B-spline curve fitting approximation (NBSC) method. The main innovation of this method is to reconstruct the flow velocity distribution fitting curve with a small amount of non-uniform feature points containing flow velocity information. First, the curvature of a whole discrete sampled data is analyzed, then the weighted threshold is set, and the sampled points that satisfy the threshold are extracted as the initial velocity distribution feature points. Next the node vectors were constructed according to the initial feature points, and the initial interpolation fitting curves are generated. Secondly, by using the relative deviation between the initial approximation curve and each sampled point, new feature points were added where the curve allowable deviation exceeded the specified tolerance, and then a new interpolation fitting curve was obtained. The above procedure was repeated until the fitting curve reached expected accuracy, thus the appropriate feature points were determined. Experimental results showed that, in the case of the same approximation deviation, the proposed NBSC method can solve the problem of under-sampling of measurement data better.

\section{Introduction}

In many engineering occasions, the velocity distribution of the fluid is required to be obtained, for the velocity of full filled pipes, through the efforts of scientific researchers, a relatively complete theory has been established. But in many cases, the fluid in the pipeline is in a state of partially filled, for example, the transport of wastewater in sewer flows, the petro chemical industries etc. [1]. The velocity distribution in a partially filled pipe is different from that of full filled pipes, the factors affecting velocity, such as wall friction and shear force, all vary with the change of the pipeline depth ratio. When the fluid flow is a partially-filled pipe laminar, the velocity distribution is relatively simple. Ng and Lawrence [2] studied the velocity of the laminar flow in partially-filled pipes with numerical simulations. The analytical expressions of laminar flow velocity in partially filled pipes are given by Guo and Meroney [3], and Fullard and Wake [4] respectively, which are verified by digital simulation. It is different from the laminar flow velocity in partially filled pipes, where the fluid is in a turbulent state, due to the influence of free surface and secondary flow in the upper part of the fluid, the velocity of the fluid is much more complex than that of the laminar flow, so the research on turbulent flow velocity distribution in partially filled pipes is a focus and it is a difficulty in the field of hydraulics.

A lot of research on the velocity of partially filled pipes have been carried out. Knight and Sterling [5], and Sterling and Knight [6] have studied the velocity in the smooth section of partially filled pipes using a Pitot-static tube. Clark and Kehler [7] researched about the mean velocity distribution of corrugated culvert using acoustic Doppler velocimetry. Using a stereoscopic particle distribution velocimetry (PIV) system, Yoon et al. [8] studied the two-dimensional velocity distribution in partially-filled circular pipes. Henry et al. [1] investigated the stream wise velocity distribution using stereoscopic particle imaging velocimetry (S-PIV) in the partially filled pipes cross-stream plane. Chiu et al. [9-13], and Marini et al. [14] analyzed the two-dimensional velocity distribution formula with Shannon entropy, which represented the observed data reasonably well in a rectangular open channel. Luo and 
Singh [15], and Cui and Singh [16] derived the partially filled pipes velocity using the Tsallis entropy, which can reasonably describe the velocity near the boundary.

These studies greatly improved the research of partially-filled pipe turbulent velocity. But all of these methods need sufficient sampled data. However, in many engineering applications, only a few measuring points can be obtained due to the measuring equipment, construction environment etc., and the known sampled data is non-uniformly distributed. Therefore, it is necessary to reasonably reconstruct the fluid velocity distribution in the case of non-uniform undersampled.

In the aspect of reconstructing the original field in the case of undersampled, a series of studies have been carried out and many theoretical methods have been also put forward, such as ordered-subsets expectation-maximization method [17], Least squares fitting algorithm $[18,19]$, NURBS curve fitting method [20, 21], B-spline curve fitting method [22, 23], etc. Because the B-spline curve method has excellent properties such as geometric invariance, convex hull, and local support, this method is often used in scientific research and engineering applications, such as data analysis and distribution reconstruction [24-27]. However, the traditional B-spline fitting curve do not directly pass through the sampled points, which leads to lower fitting accuracy and cannot truly reflect the flow velocity. Especially, when the measurement data is small, the fitting accuracy of this method is lower. How to get better flow velocity distribution reconstruction under the condition of under-sampling of measurement data, this would be the main problem to be solved in this paper.

In order to solve the previous problems, an improved non-uniform B-spline curve interpolation method called NBSC was proposed in this paper. According to the characteristics of velocity distribution, a small amount of sampling points containing velocity information are selected as featured points, and the velocity distribution curve was reconstructed according to the velocity information contained in the featured points, so as to solve the problem of under-sampling of measured data in velocity data acquisition. The main content of this method includes five aspects: (a) introduction of traditional B-spline curve method, (b) the curvature of discrete sampled points and the extraction of distribution featured points, (c) reconstruction of the node vectors, (d) adjustment of feature points, and (e) reconstruction of the flow velocity distribution.

\section{Traditional B-Spline Curve Method}

2.1. B-Spline Curve Mathematical Definition. Let $B(u)$ be the position vector along the curve as a function of the parameter $u$, a B-spline curve can be defined as

$$
B(u)=\sum_{i=0}^{k} N_{i, p}(u) P_{i} \quad u \in\left[u_{p-1}, u_{k+1}\right],
$$

where $P_{i}$ is the control points, $k+1$ is the number of control points, $p$ is the order of B-spline curve, $U=\left\{u_{0}, u_{1}, u_{2}, \ldots, u_{j}\right\}$ is the knot vector, and $N_{i, p}(u)$ is the $p$ th-order B-spline basis function, which is defined as follows:

$$
\begin{gathered}
N_{i, 0}(u)=\left\{\begin{array}{lc}
1, & u_{i} \leq u \leq u_{i+1}, \\
0, & \text { otherwise, }
\end{array}\right. \\
N_{i, p}(u)=\frac{u-u_{i}}{u_{i+p}-u_{i}} N_{i, p-1}(u)+\frac{u_{i+p+1}-u}{u_{i+p+1}-u_{i+1}} N_{i+1, p-1}(u) .
\end{gathered}
$$

The calculation process of the B-spline curve method can be summarized as follows: First, the sampled data was parameterized, then the node vector was defined according to the parameterized data. Second, the basis function can be obtained according to Equations (2) and (3). Third, the control points of the fitting curve can be calculated by using the sampling data and the basis function. Finally, with the obtaining of basis function and control points, the B-spline curve can be drawn according to Equation (1).

2.2. Uniform Node Vector with Parameter. Node vector is an important parameter in the B-spline curve fitting method. An appropriate node vector can not only ensure the curve reflecting the distribution of measurement points correctly, but also avoids matrix singularity when reverse calculating control vertexes. In any case, one needs to allocate a location parameter to each of the sampling data, then define a node vector, and finally compute the basis function and control points [20]. In this paper, the Cumulative Chord Length method [20] was used to parameterize the sampled data, then the node vectors was constructed with the parameterized sampled data.

Assuming the sampled data sequence $M=\left\{m_{0}, m_{1}\right.$, $\left.m_{2}, \ldots, m_{n}\right\}$, and chord length $l_{i}=\left\{\left|m_{i}-m_{i-1}\right|^{e}, i=1,2, \ldots, n\right\}$, in which $e=1$, then the total chord length $L=\sum_{i=1}^{n} m_{i}$, and the normalized expression of location parameter $u_{\text {norm }}$ based on the Cumulative Chord Length method can be assigned as

$$
\begin{array}{ll}
u_{\text {norm } 0}=0, & u_{\text {normt }}=1, \\
u_{\text {normi }}=\frac{1}{L} \sum_{r=1}^{i} l_{r}, & i=1,2, \ldots, n-1 .
\end{array}
$$

Let the repeatability of the node vector at both ends be $p+1$, then the relationship between control points and the sampled data is $P_{0}=m_{0}, P_{k}=m_{n}$. Assuming the domain of node vector $u \in\left[u_{p}, u_{k+1}\right]=[0,1]$, then the normalized node vector $U$ can be written as

$$
\begin{aligned}
& u_{0}=\cdots u_{p}=0, \\
& u_{k+1}=\cdots \cdot u_{k+p+1}=1, \\
& u_{i+p}=\frac{1}{p} \sum_{r=i}^{i+p+1} u_{n o r m r}, \quad i=1,2, \ldots, k-p .
\end{aligned}
$$

2.3. Construction of Control Points. The node vector $U$ of the sampling data can be calculated by Equation (5), and then the value of the basis function can be obtained by Equations (2) and (3). According to the basis function $N$ and the sampled data sequence $M$, the linear equation of the control points $P_{i}$ is given as follows

$$
\left(N^{T} N\right) P=M
$$




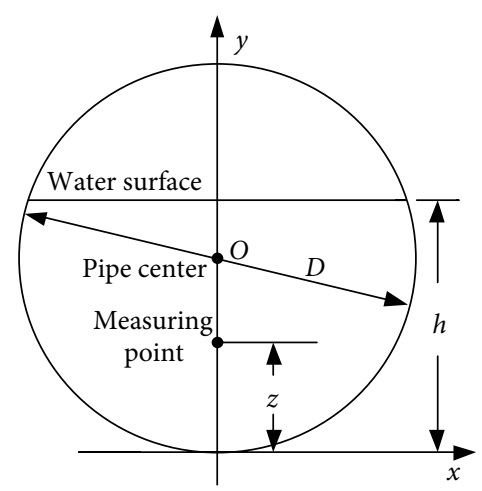

FIGURE 1: Diagram of section of measuring pipe.

The equation can be rewritten as

$$
P=\left(N^{T} N\right)^{-1} M
$$

where $T$ and -1 represent the matrix transpose. The equation can be solved with Gauss Elimination method [28], then the control points $P_{i}(i=1,2, \ldots, n-1)$ are obtained.

With the obtaining of control points $P_{i}$ and basis function $N$, the B-spline curve can be drawn according to Equation (1). Obviously, in the calculation process, the control points and basis function are the two main features of B-spline method. From the above analysis, the two parameters are mainly determined by node vector. Therefore, the merits of the node vector directly determine the fitting accuracy of the B-spline curve.

2.4. Initial B-Spline Fitting Curve. A schematic diagram of partially filled circular pipe section is shown in Figure 1, where $D$ is the diameter of the measuring pipe, and $h(0 \leq h \leq D)$ is the fluid depth from the bottom of the pipe to the water surface, and $z(0<z \leq h)$ is the vertical height from the bottom of the pipe to the position of the measuring point. A Cartesian coordinate system is set in this way such that the bottom of the pipe has the coordinate origin, and $x$ presents the transverse distance from the centerline, and $y$ presents the vertical depth from $x$ axis upward positive. According to the definition of traditional B-spline curve fitting method, the flow velocity distribution curve of the partially filled pipe flow field is shown in Figure 2.

In Figure 2, $U_{\max }$ is the maximum value of the flow velocity, where the curve obtained by the traditional B-spline curve fitting method is generally close to the velocity sampled data, but there is obvious deviation between the fitting curve and the sampled data. Moreover, all the sampled data were processed equally when the control points are inversely calculated, which makes the number of iterations increase, and greatly reduces the computational efficiency. But reducing iteration time it will cause further increase in the local deviation.

In many engineering applications, only insufficient sampled data can be obtained due to the influence of construction environment, equipment, and other factors. The B-spline curve is fitted with undersampled data, and the results are shown in Figure 3.

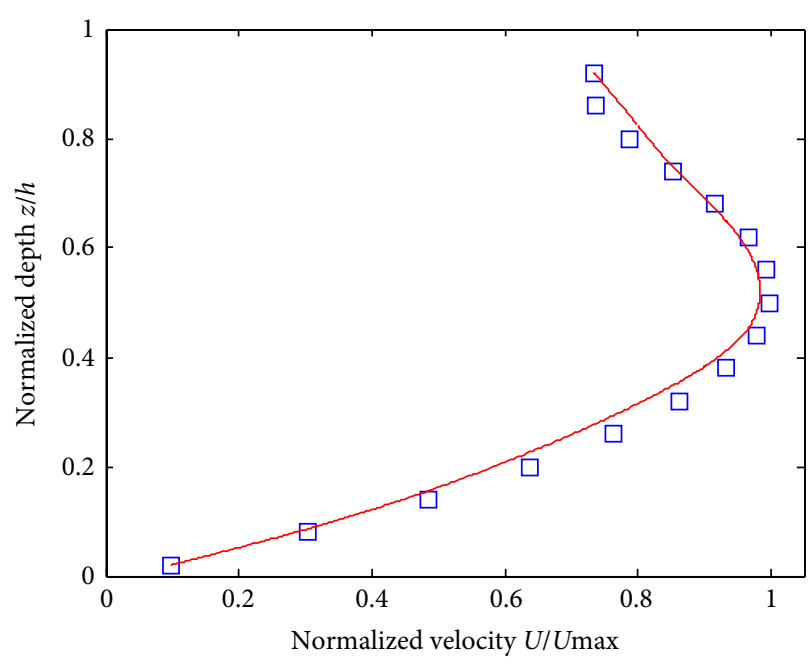

$\square$ Sampled data $\quad$ B-spline curve

Figure 2: Initial fitting curve.

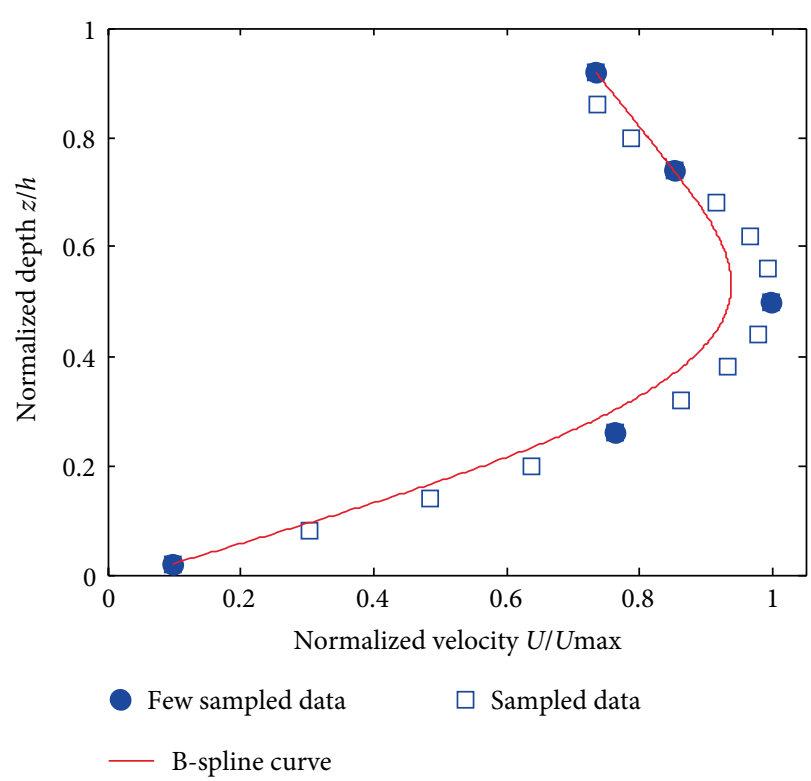

Figure 3: Few sampled data fitting curve.

From Figure 3, it is clear that the conventional B-spline curve fitting method based on a few sampled data reduces the computational complexity, however, there is a large deviation between the fitted data and the sampled data. Therefore, how to reconstruct the velocity image in the case of under-sampling is the main problem to be solved.

\section{Improved Non-uniform B-Spline Curve Method}

In many cases, only insufficient sample points can be measured. If the flow velocity information contained in these sample points can fully express the trend of the flow velocity distribution, then the B-spline curve method can be used to 
reconstruct the entire flow velocity distribution curve. Based on this idea, an improved non-uniform B-spline curve (NBSC) method was proposed. Compared with the traditional B-spline method, there are two obvious changes in this method. First, some sampled data were extracted as feature points, which have important influence on the shape of approximation curve. Second, the non-uniform node vectors were reconstructed with these extracted feature points. The basis function and control points were updated with the new node vector. With the obtaining of new control points the new fitting curve were drawn. Obviously, the key to this method is to reasonably select feature points containing flow velocity information. In this paper, the curvature distribution method is used to determine the feature points of the fitting curve.

3.1. The Curvature of Discrete Points. In comparison with several methods of algorithm for curvature [29-31], the curvature distribution was solved with Local Estimation method in this paper. In the Local Estimation method, the parametric polynomials $S(m)$ are established by using the front and back three points of the measured points, then the curvature of the sampled point $m_{i}$ in the data sequence can be expressed as

$$
K_{i}=\frac{\left\|S^{\prime}\left(m_{i}\right) \times S^{\prime \prime}\left(m_{i}\right)\right\|}{\left\|S^{\prime}\left(m_{i}\right)\right\|^{2}} \quad i=0,1, \ldots, n,
$$

where $S^{\prime}\left(m_{i}\right)$ represent the first derivative of the curve at parameter $m_{i}, S^{\prime \prime}\left(m_{i}\right)$ represent the second derivative, and the magnitude of the curvature reflects the variation degree of flow velocity.

3.2. Selection of Unbalanced Feature Points. According to Formula (8), the curvature of flow velocity at each sampled point on the central line of the pipeline can be obtained, the result is shown in Figure 4, where the abscissa is the height of the measuring point from the bottom of the tube, and the longitudinal coordinate is the curvature of the flow velocity at each measuring position.

Obviously, the curvature value of the curve is small where the velocity varies smooth, while the curvature value of the curve is relatively large the velocity varies sharply. Therefore, in the sampled data sequence, the principle of selecting feature points are as follows:

(1) The sampled point with maximum curvature $K_{\max }$ is the featured point.

(2) The first sampled point $m_{0}$ and the end sampled point $m_{n}$ are the featured points.

(3) The sampled points higher than the preset curvature threshold $K_{\text {pre }}$ are the featured points. It should be noted that the curvature threshold is too high to reflect the characteristics of the curve. On the contrary, reducing the threshold will result in a large amount of calculation and lower computational efficiency. Therefore, the curvature weighted average $w K_{a v}$ is used as the preset threshold in this paper, where $K_{a v}$ is the average of curvature and $w$ is the weight.

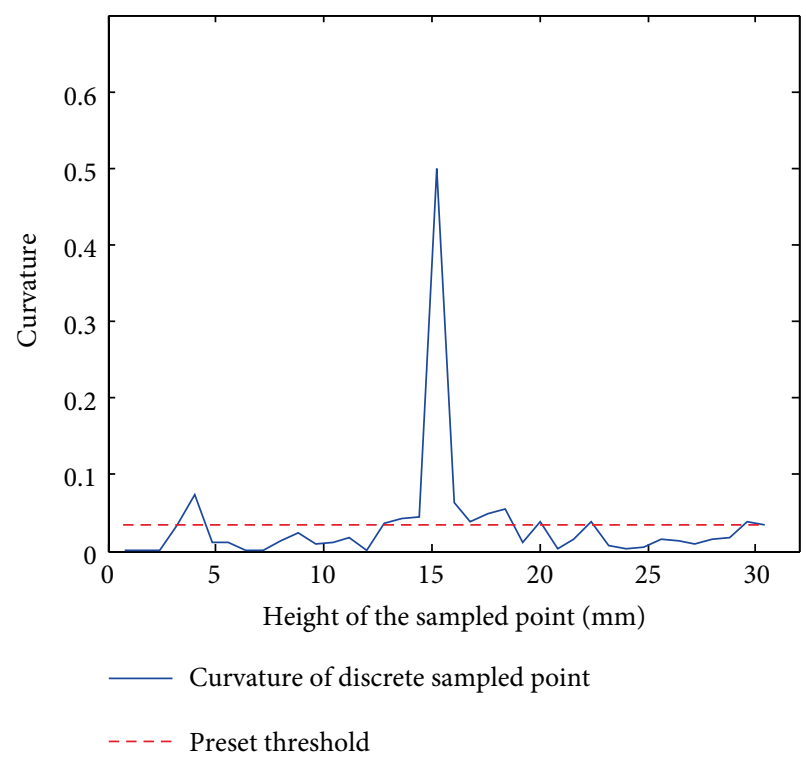

FIGURE 4: The distribution of curvature.

3.3. Restructuring of the Non-uniform Node Vector. According to the mentioned principle of selecting unbalanced feature points, the sampled points $M_{f e a}\left\{m_{f e a 0}, m_{f e a 1}, \ldots, m_{\text {feat }}\right\}$ are selected as feature points, in which the first point $m_{\text {fea } 0}=m_{0}$, and the end point $m_{\text {feat }}=m_{n}$. The chord length is redefined as $l_{j}=\left\{\left|m_{f e a j}-m_{f e a(j-1)}\right|^{e}, j=1,2, \ldots, t\right\}, e=1$, then the location parameter $\hat{u}_{\text {norm }}$ and the non-uniform node vector $\widehat{U}$ can be rewritten as

$$
\begin{aligned}
& \hat{u}_{\text {norm } 0}=0, \quad \hat{u}_{\text {normt }}=1, \\
& \hat{u}_{n o r m j}=\frac{1}{L} \sum_{r=1}^{j} l_{r}, \quad j=1,2, \ldots, t-1,
\end{aligned}
$$

and

$$
\begin{aligned}
\hat{u}_{0} & =\cdots \bar{u}_{p}=0, \\
\hat{u}_{t+1} & =\cdots \bar{u}_{t+p+1}=1, \\
\hat{u}_{j+p} & =\frac{1}{p} \sum_{r=j}^{j+p+1} \widehat{u}_{n o r m r}, \quad j=1,2, \ldots, t-p,
\end{aligned}
$$

where $\hat{u}_{\text {normr }}$ is the quadratic normalized parameter value of the featured points. Then the basis function and the control points were updated according to Equations (3) and (7). With the obtaining of new control points the new fitting curve were drawn.

3.4. Quadratic Optimization of the Fitting Curve. For the initial fitting curve, if the local approximation deviation exceeds the maximum allowable deviation $\varepsilon$, some new feature points should be added appropriately to improve the local fitting accuracy. According to the proposed method, the fitting curve $B(u)$ based on discrete sampled points can be obtained, and the relationship between the fitting curve and sampled data are as follows

$$
\max _{i=1,2, \ldots, n} \frac{d\left(B\left(u_{i}\right), m_{i}\right)}{m_{i}}=g_{i} \leq \varepsilon,
$$




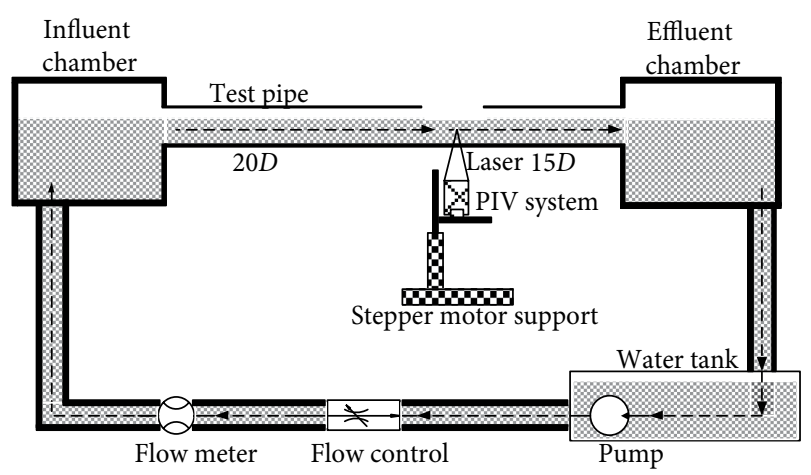

FIGURE 5: Schematic diagram of the test equipment.

where $\varepsilon$ is the maximum relative tolerance of deviation, $d\left(B(u), m_{i}\right)$ is the distance from measured data point to the nearest point of the fitting curve, i.e., $d\left(B(u), m_{i}\right)=\min \left|B(u)-m_{i}\right|$. If $g_{i}>\varepsilon$, the corresponding sampled data point $m_{i}$ is the new featured point, and the node vector was reconstructed again with the non-uniform node vector construction method, then the approximation curve of quadratic optimization is obtained.

\section{Experiment and Discussion}

4.1. Experimental Measurement. The schematic test equipment is shown in Figure 5. The Particle Image Velocimetry (PIV) system was adopted to measure the fluid velocity of a single point, and the measurement accuracy was less than $0.5 \%$ in theory. The diameter of the transparent acrylic test pipe is $D=0.04 \mathrm{~m}$. In order to ensure the fully developed flow profiles in the measuring section, the distance between the pipe inlet and the observation point are $20 \mathrm{D}$, and the distance between the pipe outlet and the measuring point are $15 \mathrm{D}$. The hydraulic slope of the test pipe is $S=0.0033$, the pipe wall was hypothesized to be hydro-dynamically smooth, and the roughness coefficient is $n_{r c}=0.0085$. A laser light emitted from a $30 \mathrm{~mJ} \mathrm{Nd:YAG} \mathrm{laser} \mathrm{to} \mathrm{illuminate} \mathrm{the} \mathrm{test} \mathrm{point,} \mathrm{and} \mathrm{the}$ interval of laser pulse is $20 \mathrm{~ns}$. Some glass spheres with diameter of $40 \mu \mathrm{m}$ were injected in order to speed the flow. The flow of the fluid depends on the drive of the pump power, and there is no device for stabilizing the fluid flow in the measurement system. The flow depth $H$ varies from $42 \%$ to $79 \%$ of $D$.

4.2. Experimental Results. The streamwise mean velocity profiles at pipe vertical bisector was measured by the PIV on the cross-sectional plane for $42 \%, 50 \%, 62 \%, 70 \%$, and $79 \%$ flow depth. In the measurement sequence, the uncertainty of the $i$-th measurement point can be expressed as.

$$
S_{u}=\sqrt{\frac{\sum_{j=1}^{k}\left(m_{i j}-\bar{m}_{i}\right)}{k(k-1)}} \quad i=0,1, \ldots, n,
$$

where $k$ is the number of repeated measurements of a single measurement point, and $\bar{m}_{i}$ is the average value of repeated measurements. According to Equation (12), the uncertainty of measurement of all measurement points were calculated, the uncertainty ranges from 0.00012 to 0.00043 . In the vicinity of the bottom of the pipeline, the tracer particles collide with the pipe wall under the action of gravity, resulting in disordered motion. This disordered motion will affect the

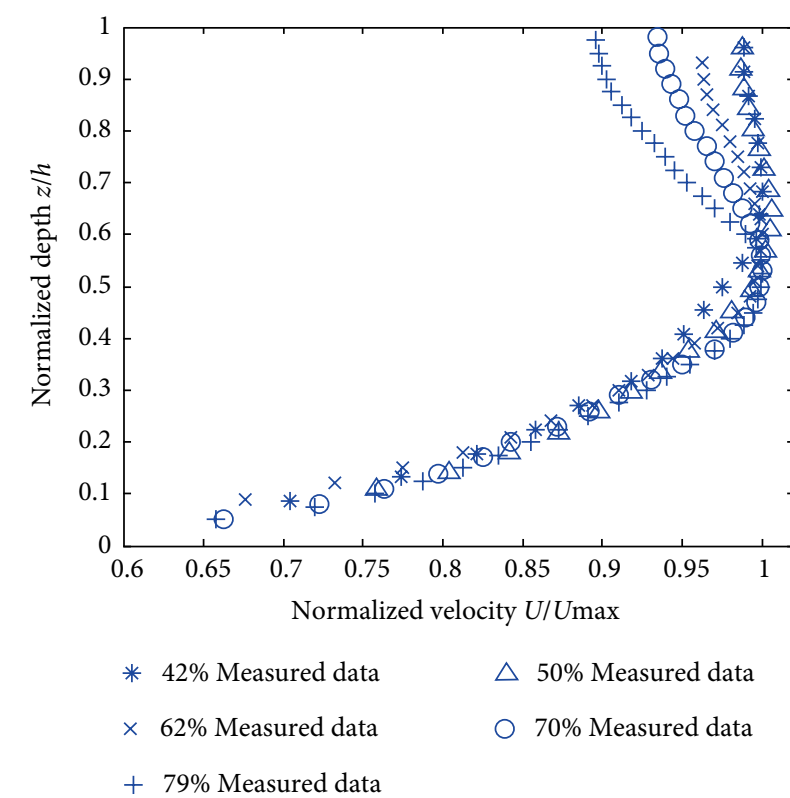

Figure 6: Velocity distribution profile.

uncertainty of measurement, resulting in greater uncertainty closer to the bottom of the pipeline, and vice versa.

The measurement results are shown in Figure 6. Where $h$ is the flow depth, $z$ is the distance from the measuring position to the pipe bottom, and $U_{\max }$ is the maximum velocity on the central section of the pipeline.

From Figure 6, the position of maximum velocity $U_{\max }$ in the pipeline is below the free surface, and the position varies with different water depth, which is consistent with the measurement result of literature [1].

4.3. Comparisons and Discussions. According to the proposed NBSC method, the average velocity along the pipeline center line is processed, and the approximation curve of velocity profile at different flow depths are obtained. Under the condition of relative deviation less than $1 \%$, the NBSC method was compared with the conventional B-spline curve method, and the results are shown in Figure 7. These data are presented using both $U$ and $U_{\max }$ as the normalized velocity scale, the height of measuring point $z$ and the flow depth $h$ are used as normalized length scales.

Figure 7 shows the comparison profiles of partially filled pipe fluid for flow depths of $42 \%, 50 \%, 62 \%, 70 \%$, and $79 \%$ respectively. As can be seen

(1) The number of sampling points required for the improved B-spline curve fitting method is far less than that required by the traditional method. When the flow depth of the pipeline are 50\% and 79\% respectively, the appropriate fitting curve can be obtained only with four measuring points, and when the flow depth are $42 \%, 62 \%$, and $70 \%$ respectively, only one feature point needs to be added. The workload of the measurement is greatly reduced.

(2) Except for the two feature points at the head end and the end point, the other feature points are located at a place where the flow velocity changes relatively, and the distribution of feature points are non-uniform. 


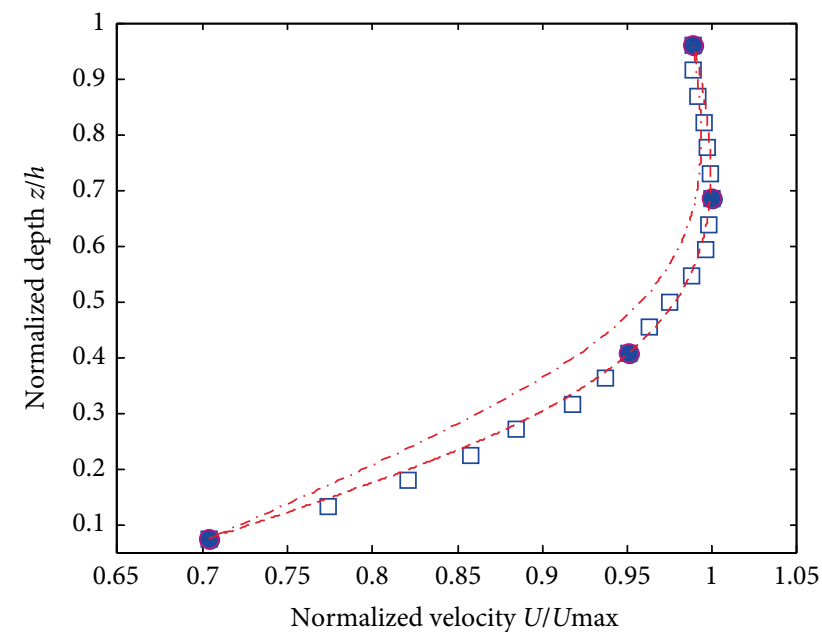

$\square$ Measured data

Orginal feature points

- - - First optimized fitting curve _. - Original curve

(a)

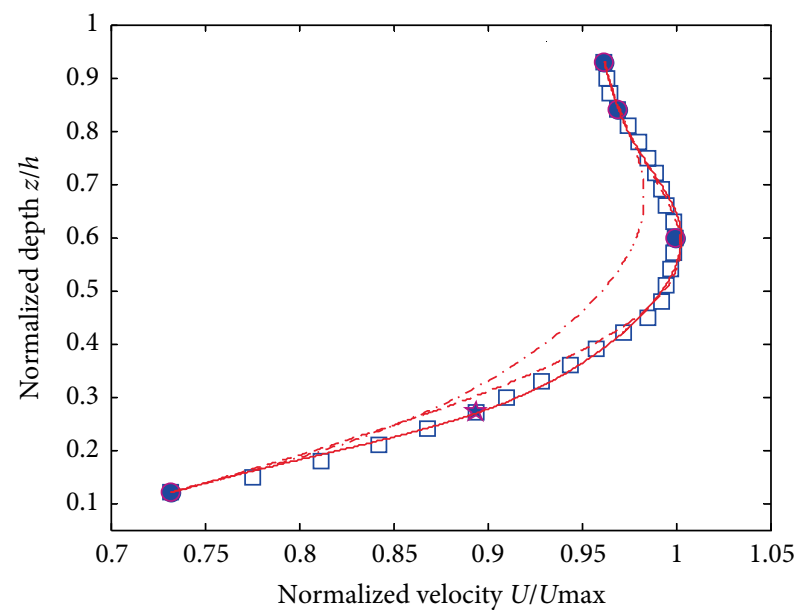

$\square$ Measured data

- Orginal feature points

$\star$ New feature points

- - - First optimized fitting curves

_ Second optimized fitting curve

...- Original curve

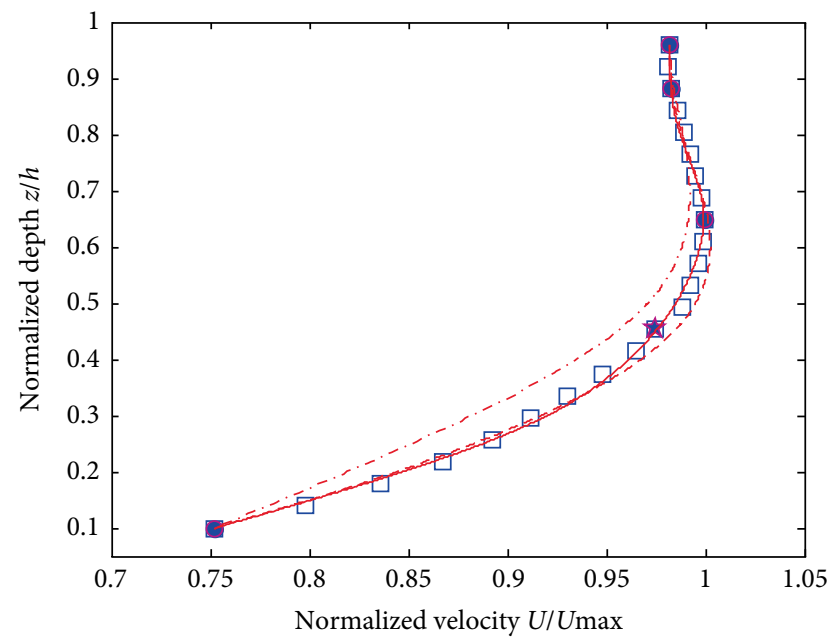

$\square$ Measured data

- Orginal feature points

$\star$ New feature points

- - - First optimized fitting curve

— Second optimized fitting curve

... Original curve

(b)

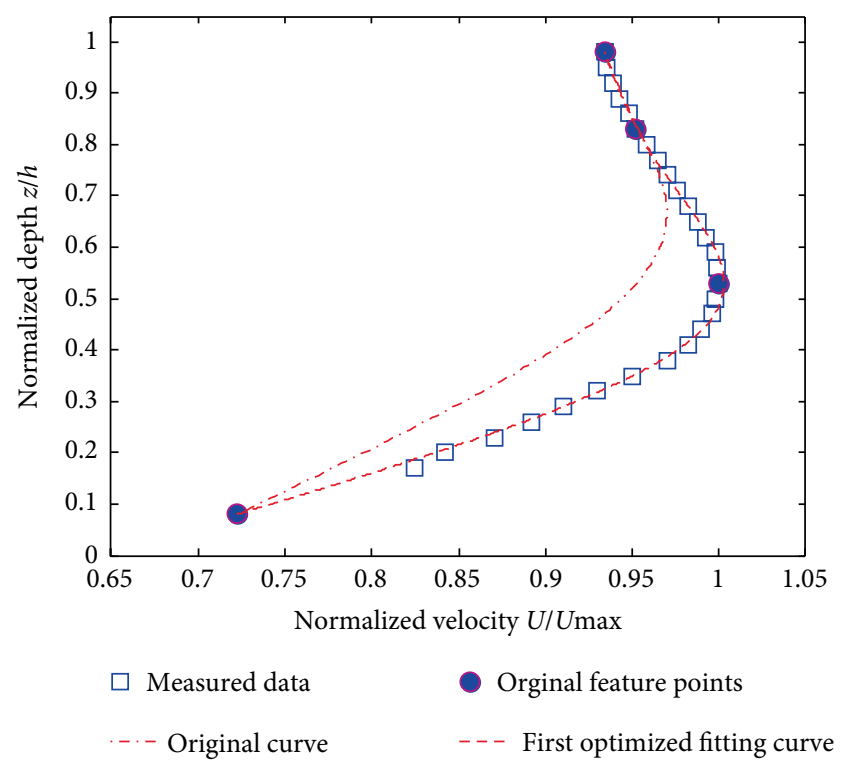

(d)

(c)

FIgure 7: Continued. 


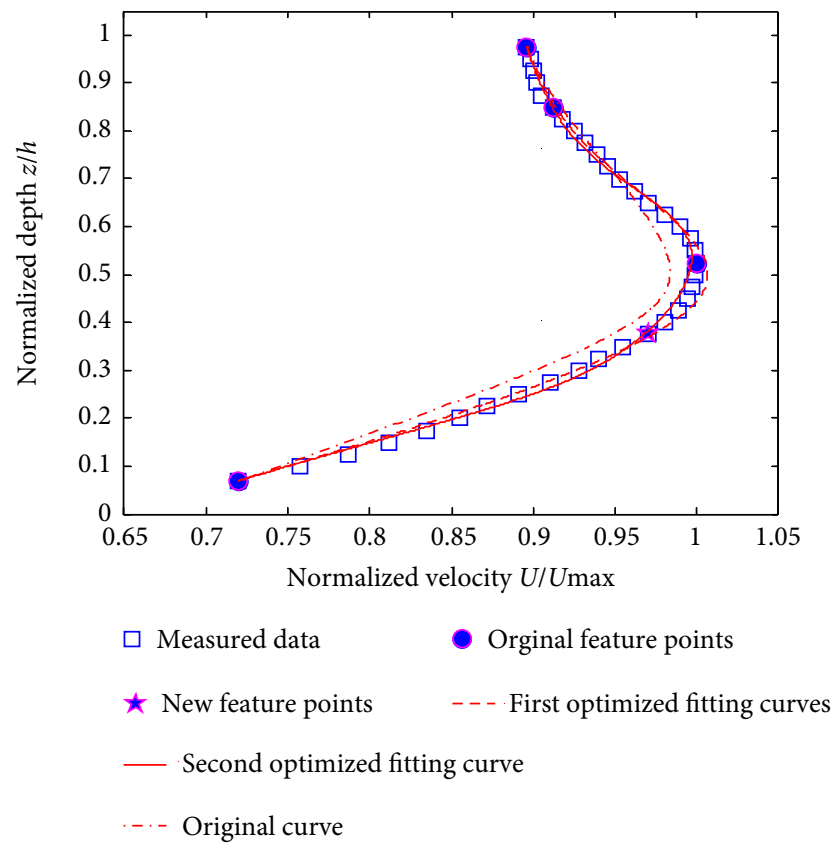

(e)

FIGURE 7: Comparisons between traditional B-spline curve method and NBSC method (a) Flow depth ratio $h / D=42 \%$. (b) Flow depth ratio $h / D=50 \%$. (c) Flow depth ratio $h / D=62 \%$. (d) Flow depth ratio $h / D=70 \%$. (e) flow depth ratio $h / D=79 \%$.

The measured object can be measured more specifically by using the proposed method in this paper.

(3) In the case of few feature points, there are a large deviation between the traditional B-spline fitting curve and the measured data. The approximation curve obtained by the first-order optimized B-spline curve fitting method can be better described as the distribution of flow velocity, but there is an obvious deviation in the local region. This deviation can be effectively reduced by using the quadratic optimization B-spline curve fitting method. This means that in a flow field where the flow velocity changes more drastically, the fitting accuracy can be improved by appropriately increasing the feature points.

(4) Using the method proposed in this paper, the flow velocity distribution can be reconstructed with only a small amount of feature points and water depth ratio. Under the condition of relative deviation less than $1 \%$, using the improved B-spline curve fitting method, the one-dimensional velocity distribution profile can be reconstructed with at most five feature points.

\section{Conclusion}

The velocity distribution in partially filled pipe is researched with non-uniform B-spline curve fitting method. A B-spline curve approximation algorithm based on few feature points is proposed, which mainly includes feature point extraction algorithm based on discrete curvature analysis, and feature point adjustment algorithm based on deviation analysis. The conclusion are as follows:
(1) When the number of measurement points are the same, when compared with the traditional B-spline curve method, the proposed method has higher fitting precision. The deviation of $\mathrm{B}$-spline approximation curve determined by feature points is obviously smaller than that of the traditional method.

(2) When the fitting accuracy is the same, the workload of the proposed method is much lesser than that of the traditional B-spline curve method. The number of feature points obtained by extraction algorithm is obviously less than that of original measurement points, which reduces the computational complexity and improves the efficiency.

(3) When the flow velocity changes drastically, more feature points are required, and when the flow velocity changes slowly, fewer feature points are required. This distribution law allows the measurements to be targeted more.

(4) The method presented in this paper is easy to understand and apply to engineering applications. The analysis shows that this method has important practical significance for engineering applications. It can be used in steel smelting, aerospace, and other harsh environments, and can also be used in mold design, garment customization, big data analysis, etc., which can reduce the workload of design.

\section{Data Availability}

The data used to support the findings of this study are available from the corresponding author upon request. 


\section{Conflicts of Interest}

The authors declare that there are no conflicts of interest regarding the publication of this paper.

\section{Acknowledgments}

This work was supported by the Science and Technology Project of Education Department of the Guangdong Province, China (2017GKTSCX079), and Science and Technology Project of Education Department of the Jiangxi Province, China (GJJ151069).

\section{References}

[1] H. C.-H. Ng, H. L. F. Cregan, J. M. Dodds, R. J. Poole, and D. J. C. Dennis, "Partially filled pipes: experiments in laminar and turbulent flow," Journal of Fluid Mechanics, vol. 848, pp. 467-507, 2018.

[2] T. S. Ng, C. J. Lawrence, and G. F. Hewitt, "Gravity-driven laminar flow in a partially-filled pipe," Chemical Engineering Research and Design, vol. 79, no. 4, pp. 499-511, 2001.

[3] J. K. Guo and R. N. Meroney, "Theoretical solution for laminar flow in partially-filled pipes," Journal of Hydraulic Research, vol. 51, no. 4, pp. 408-416, 2013.

[4] L. A. Fullard and G. C. Wake, "An analytical series solution to the steady laminar flow of a Newtonian fluid in a prtially filled pipe, including the velocity distribution and the dip phenomenon," IMA Journal of Applied Mathematics, vol. 80, no. 6, pp. 1890-1901, 2015.

[5] D. W. Knight and M. Sterling, "Boundary shear in circular pipes running partially full," Journal of Hydraulic Engineering, vol. 126, no. 4, pp. 263-275, 2000.

[6] M. Sterlin and D. W. Knight, "Resistance and boundary shear in circular conduits with flat beds running part full," Proceedings of the ICE-Water and Maritime Engineering, vol. 142, no. 4, pp. 229-240, 2000.

[7] S. P. Clark and N. Kehler, "Turbulent flow characteristics in circular corrugated culverts at mild slopes," Journal of Hydraulic Research, vol. 49, no. 5, pp. 676-684, 2011.

[8] J. Yoon, J. Sung, and M. H. Lee, "Velocity profiles and friction coefficients in circular open channels," Journal of Hydraulic Research, vol. 50, no. 4, pp. 304-311, 2012.

[9] C. L. Chiu, "Entropy and 2-D velocity distribution in open channels," Journal of Hydraulic Engineering, vol. 114, no. 7, pp. 738-756, 1988.

[10] C. L. Chiu, "Application of entropy concept in open channel flow study," Journal of Hydraulic Engineering, vol. 117, no. 5, pp. 615-628, 1991.

[11] C. L. Chiu and C. A. Said, "Maximum and mean velocities and entropy in open-channel flow," Journal of Hydraulic Engineering, vol. 121, no. 1, pp. 26-35, 1995.

[12] C. L. Chiu, S. M. Hsu, and N. C. Tung, "Efficient methods of discharge measurements in rivers and streams based on the probability concept," Hydrology Processes, vol. 19, no. 20, pp. 3935-3946, 2005.

[13] C. L. Chiu and S. H. Hsu, "Probabilistic approach to modeling of velocity distributions in fluid flows," Journal of Hydrology, vol. 316, no. 1-4, pp. 28-42, 2006.
[14] G. Marini, G. D. Martino, N. Fontana, M. Fiorentino, and V. P. Singh, "Entropy approach for $2 \mathrm{D}$ velocity distribution in-open channel flow," Journal of Hydraulic Research, vol. 49, no. 6, pp. 784-790, 2011.

[15] H. Luo and V. P. Singh, "Entropy theory for two-dimensional velocity distribution," Journal of Hydraulic Engineering, vol. 16, no. 4, pp. 303-315, 2011.

[16] H. J. Cui and V. P. Singh, "Two-dimensional velocity distribution in open channels using the Tsallis entropy," Journal of Hydraulic Engineering, vol. 18, no. 3, pp. 331-339, 2013.

[17] L. J. Xu, X. M. Li, and F. Dong, "Optimum estimation of the mean flow velocity for the multi-electrode inductance flowmeter," Measurement Science and Technology, vol. 12, no. 8, pp. 1139-1146, 2001.

[18] W. Gander, G. H. Golub, and R. Strebel, "Least-squares fitting of circles and ellipses," BIT Numerical Mathematics, vol. 34, no. 4, pp. 558-578, 1994.

[19] C. M. Shakarji, "Least-squares fitting algorithms of the NIST algorithm testing system," Journal of Research of the National Institute of Standards and Technology, vol. 103, no. 6, pp. 633-641, 1998.

[20] L. Piegl and W. Tiller, The Nurbs Book, Springer-Verlag, New York, N.Y., USA, 2nd edition, 1997.

[21] E. Ulker, "Nurbs curve fitting using artificial immune system," Internal Journal of Innovative Computing, Information and Control, vol. 8, no. 4, pp. 2875-2887, 2012.

[22] E. Saux, "B-spline curve fitting: application to cartographic generalization of maritime Lines," in 8th International Conference on Computer Graphics and Visualization (GraphiCon'98), pp. 196-2031998.

[23] X. N. Li, M. J. Xu, X. Q. Lv, and K. Fu, "A study of the transport of Marine pollutants using adjoint method of data assimilation with method of characteristics," Advances in Mathematical Physics, vol. 2018, Article ID 7408263, 14 pages, 2018.

[24] H. Park, "An error-bounded approximate method for representing planar curves in B-splines," Computer Aided Geometric Design, vol. 21, no. 5, pp. 479-497, 2005.

[25] H. Park and J. H. Lee, "B-spline curve fitting based on adaptive curve refinement using dominant points," Computer-Aided Design, vol. 39, no. 6, pp. 439-451, 2007.

[26] L. Piegl and W. Tiller, "Least-squares B-spline curve approximation with arbitrary end derivatives," Engineering with Computers, vol. 16, no. 2, pp. 109-116, 2000.

[27] G. Y. He, C. H. Liu, Y. C. Sang, and X. Sun, "The calculation of roughness uncertainty by fitting B-spline filter assessment middle lines," Mathematical Problems in Engineering, vol. 2019, Article ID 6913215, 10 pages, 2019.

[28] W. S. Li, S. H. Xu, G. Zhao, and L. P. Goh, "Adaptive knot placement in B-spline curve approximation," Computer Aided Design, vol. 37, no. 8, pp. 791-797, 2005.

[29] G. H. Liu, Y. S. Wong, Y. F. Zhang, and H. T. Loh, "Adaptive fairing of digitized point data with discrete curvature," Computer Aided Design, vol. 34, no. 4, pp. 309-320, 2002.

[30] T. Takami and H. Haseqawa, "Curvature distribution of chaotic quantum systems: universality and nonuniversality," Physical Review Letters, vol. 68, no. 4, pp. 419-422, 1992.

[31] X. T. Zhong, J. R. David, B. Hossein, and G. S. Rohrer, "The five-parameter grain boundary curvature distribution in an austenitic and ferritic steel," Acta Materialia, vol. 123, pp. 136-145, 2017. 


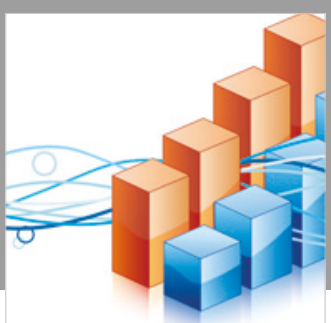

Advances in

Operations Research

\section{-n-m}
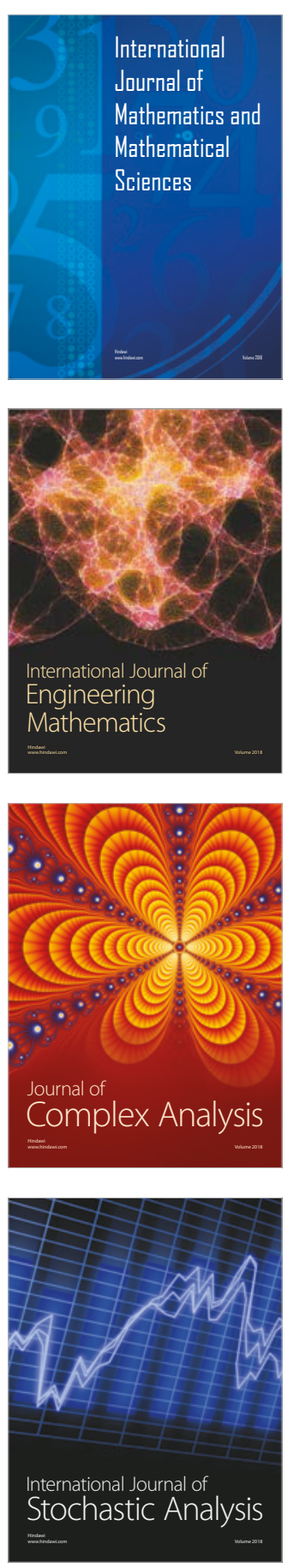
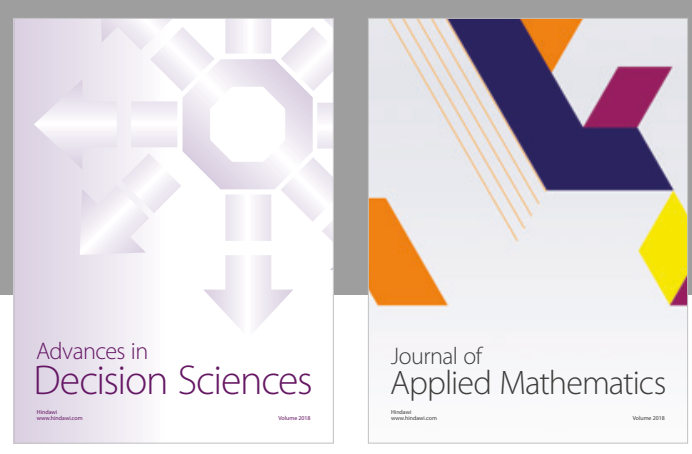

Journal of

Applied Mathematics
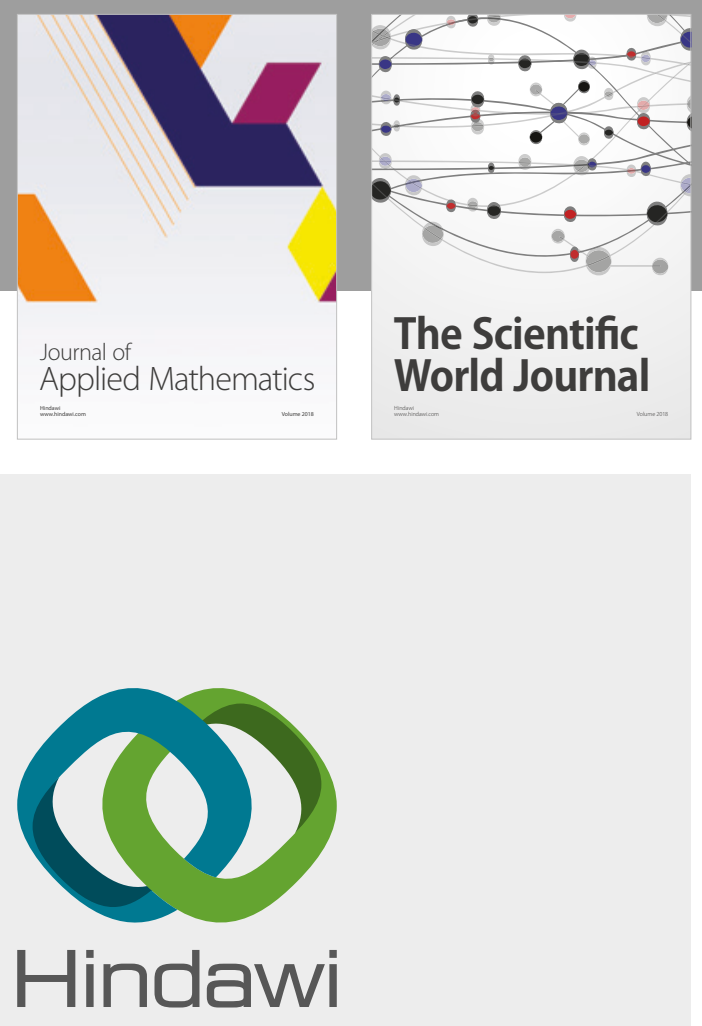

Submit your manuscripts at

www.hindawi.com

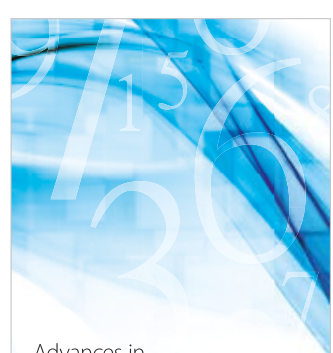

Advances in
Numerical Analysis
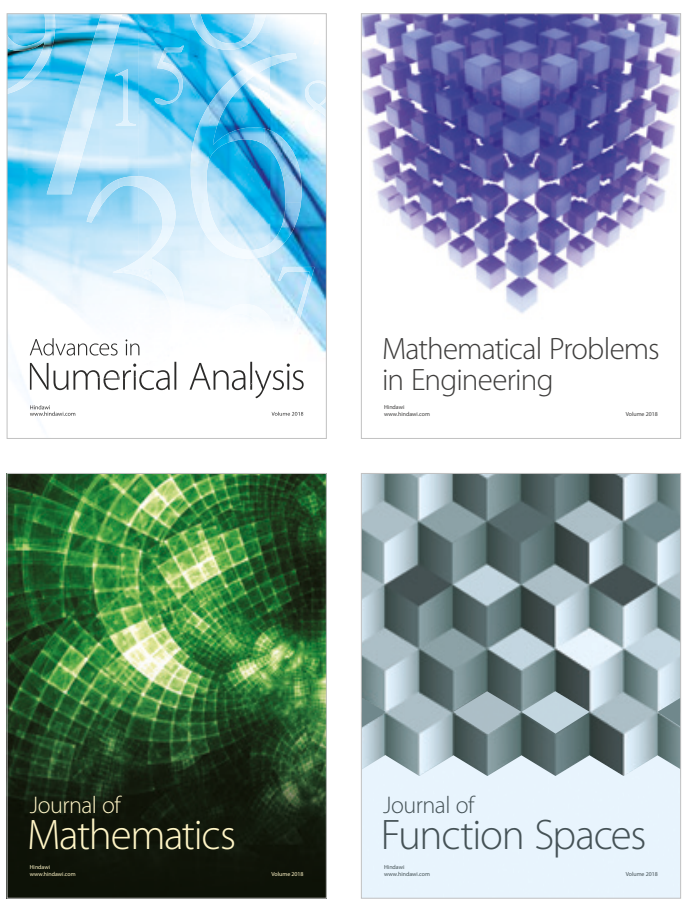

Mathematical Problems in Engineering

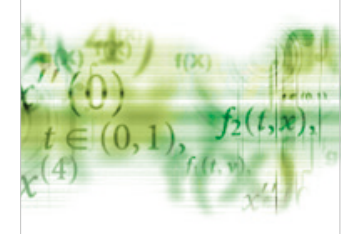

International Journal of

Differential Equations

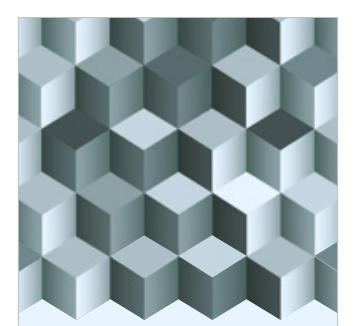

Journal of

Function Spaces
The Scientific

World Journal

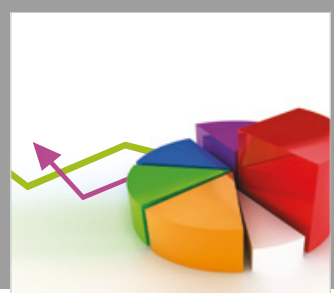

Journal of

Probability and Statistics
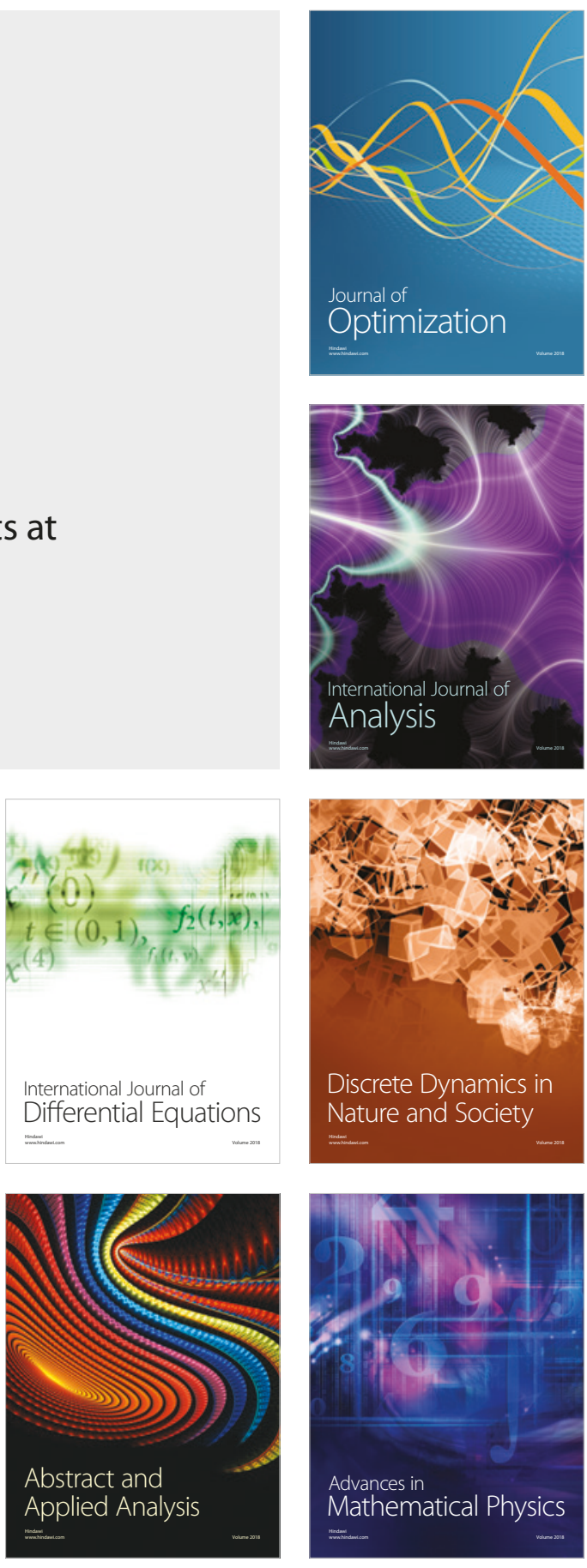